\title{
BMJ Open Predicting prostate cancer progression: protocol for a retrospective cohort study to identify prognostic factors for prostate cancer outcomes using routine primary care data
}

Samuel W D Merriel, ${ }^{1}$ Margaret T May, ${ }^{2}$ Richard M Martin ${ }^{2}$

To cite: Merriel SWD, May MT, Martin RM. Predicting prostate cancer progression: protocol for a retrospective cohort study to identify prognostic factors for prostate cancer outcomes using routine primary care data. BMJ Open 2018;8:e019409. doi:10.1136/ bmjopen-2017-019409

- Prepublication history for this paper is available online. To view these files, please visit the journal online (http://dx.doi. org/10.1136/bmjopen-2017019409).

Received 31 August 2017 Revised 27 November 2017 Accepted 8 December 2017

Check for updates

${ }^{1}$ Centre for Academic Primary Care, University of Bristol, Bristol, UK

${ }^{2}$ Department of Population Health Sciences, University of Bristol, Bristol, UK

Correspondence to Dr Samuel W D Merriel; sam.merriel@bristol.ac.uk

\section{ABSTRACT}

Introduction Prostate cancer is the most common cancer in men in the UK, with nearly 40000 diagnosed in 2014; and it is the second most common cause of male cancer-related mortality. The clinical conundrum is that most men live with prostate cancer rather than die from it, while existing treatments have significant associated morbidity. Recent studies have shown very low mortality rates ( $1 \%$ after a median of 10 -year followup) and no treatment-related reductions in mortality, in men with localised prostate cancer. This study will identify prognostic factors associated with prostate cancer progression to help differentiate aggressive from more indolent tumours in men with localised disease at diagnosis, and so inform the decision to adopt conservative (active surveillance) or radical (surgery or radiotherapy) management strategies.

Methods and analysis The Clinical Practice Research Datalink (CPRD) contains 57318 men who were diagnosed with prostate cancer between 1 January 1987 and 31 December 2016. These men will be linked to the Office for National Statistics (ONS) and the National Cancer Registration and Analysis Service registry databases for mortality, TNM stage, Gleason grade and treatment data. Men with a diagnosis date prior to 1 January 1987 and men with lymph node or distant metastases at diagnosis will be excluded. A priori determined prognostic factors potentially associated with prostate cancer mortality, the end point of cancer progression, will be measured at baseline, and the participants followed through to development of cancer progression, death or the end of the follow-up period (31 December 2016). Cox proportional hazards regression will be used to estimate crude and mutually adjusted HRs. Mortality risk will be predicted using flexible parametric survival models that can accurately fit the shape of the hazard function.

Ethics and dissemination This study protocol has approval from the Independent Scientific Advisory Committee for the UK Medicines and Healthcare products Regulatory Agency Database Research (protocol 17_041). The findings will be presented in peer-reviewed journals and local CPRD researcher meetings.

\section{Strengths and limitations of this study}

- The study cohort is drawn from the Clinical Practice Research Datalink (CPRD), a large, representative UK primary care dataset, with linked cancer registry and Office for National Statistics (ONS) data.

> Predicting cancer progression is a more clinically useful outcome than simply detecting localised disease in patients with a disease that is often indolent and slow-growing.

- Flexible parametric modelling will be used to control for the effects of intermediate variables and prognostic factor combinations will be used.

- Cancer registry data and ONS mortality data are not available for the full study period, and CPRD data may not be complete.

\section{INTRODUCTION}

Prostate cancer is the most commonly diagnosed cancer in men, and the second most commonly diagnosed cancer in the UK. A total of 39741 new cases of prostate cancer were diagnosed in England in 2014, with an age-standardised incidence rate of 177.8 per 100000 men. ${ }^{1}$ In the same year, there were approximately 11300 prostate cancer deaths in the UK, making prostate cancer the second most common cause of cancer death in men. Nevertheless, survival rates for prostate cancer are relatively high compared with other cancer types. The overall 5-year age-standardised net survival for men with prostate cancer in England was 83.6\%, and the predicted 10-year survival for men in England diagnosed with prostate cancer in 2015 is $79.9 \%$. $^{2}$ This suggests that many men diagnosed with prostate cancer have indolent disease. A key clinical conundrum relates to distinguishing men with slow-growing tumours that could be managed conservatively with active monitoring from more 
aggressive, potentially fatal disease that may require more radical intervention.

Prostate cancer can be detected in men in different ways. General practitioners (GPs) need to consider the possibility of prostate cancer in men presenting with lower urinary tract symptoms, erectile dysfunction or visible haematuria. Asymptomatic men may also be found to have raised prostate-specific antigen (PSA) levels and need to be referred for further investigation. ${ }^{3}$ However, the use of PSA as a screening and prognostic biomarker remains controversial, ${ }^{45}$ and $\mathrm{GPs}^{6-9}$ and patients ${ }^{10}$ have mixed views about its utility in informing investigation and treatment decisions for prostate cancer. Other screening methods for predicting prostate cancer severity have been tested, such as the Stockholm-3 (STHLM-3) model, ${ }^{11}$ which did not look at risk of progression and relies on genetic biomarkers that are not readily available in primary care at this time.

The Bristol-based Protec $T$ multicentre trial randomised men with clinically localised prostate cancer to either active monitoring, radical surgery (prostatectomy) or radical radiotherapy. After a median of 10-year follow-up there was no difference in prostate cancer mortality. Overall, the 10-year mortality rates were very low $(1 \%)$, and men randomised to active monitoring were at an increased risk of clinical progression and development of metastatic disease (22.9 per 1000 person years follow-up) compared with the radical treatment arms (8.9 and 9 per 1000 person years, respectively). ${ }^{12}$ Men receiving surgery or radiotherapy reported more adverse effects on urinary, sexual and bowel function compared with the active monitoring cohort. ${ }^{13}$ This was broadly consistent with other studies of prostate cancer treatment. ${ }^{14-18}$ Identifying factors associated with prostate cancer progression may help determine the risk for men having more aggressive prostate cancer, and inform shared decision-making about whether to undergo radical treatments or choose active monitoring.

Cancer progression is well defined in cancer treatment trials, following the widely used RECIST criteria. $^{19}$ However, the concept of cancer progression in prognostic studies is much less well defined or consistently applied. The Protec $T$ trial defined prostate cancer progression as the occurrence of any of the following events: evidence of metastasis, development of T3/T4 disease, commencing long-term androgen therapy, ureteric obstruction, rectal fistula and new need for catheter. ${ }^{12}$ Several prognostic factors have been identified that may be associated with prostate cancer mortality, the endpoint of prostate cancer progression. These include demographic, ${ }^{20}$ genetic, ${ }^{21}$ physiological, ${ }^{22} 23$ comorbidity, ${ }^{24-27}$ lifestyle, ${ }^{28-33}$ biochemical $^{3435}$ and medication ${ }^{3637}$ factors. The strength of evidence for these prognostics factors varies and for many others it is conflicting. ${ }^{38-44}$

Primary care medical records contain a wealth of information on a patient's medical history, medications, family history and investigation results. ${ }^{45}$ The Clinical Practice Research Datalink (CPRD $)^{46}$ is a large UK primary care research database representative of the general population, with links to many other relevant healthcare data registries and Office for National Statistics (ONS) data. This information is already used for many risk prediction tools in primary care settings to predict outcomes and inform treatment decisions. Examples include QCancer, ${ }^{47}$ which predicts a patient's absolute risk of future cancer diagnosis. To date there are no risk prediction tools for cancer progression used in clinical practice.

This study aims to establish which risk factors are associated with prostate cancer progression using primary care medical records data. These findings, in combination with metabolomic and genomic data, will inform the development of a clinical risk prediction model for the progression of prostate cancer following diagnosis.

\section{METHODS AND ANALYSIS}

Within the CPRD dataset, at least 57318 men had a diagnosis of prostate cancer made between 1 January 1987 and 31 December 2016, of whom 22080 have a recorded date of death. These men will form the basis of the study cohort. Additional mortality, staging (TNM and Gleason grade) and treatment data will be obtained by using each man's National Health Service (NHS) number to link them to the ONS (available from 1 January 1998) and National Cancer Registration and Analysis Service (available from 1 January 1990) databases. The index date will be the date the diagnosis of prostate cancer was first entered into the primary care medical record. From this date, the men will be followed until the date of their death, the development of prostate cancer progression or the end of the cohort period, whichever is later. Men with a diagnosis date prior to 1 January 1987 and men with lymph node or distant metastases at diagnosis will be excluded from the analysis.

Each of the hypothesised prognostic factors for prostate cancer mortality identified a priori (see table 1) will be recorded as an 'exposure' if it is entered into the patient record at the study baseline (index date), or recorded before the diagnosis of prostate cancer is entered. Continuous variables, such as height, weight and the biochemical markers, will be measured according to the most recent result prior to the coding of a diagnosis of prostate cancer within the study time period. Genetic factors, lifestyle exposures, medications and comorbidities will be considered in a binary manner in relation to their presence or absence at the index date. Missing data will be controlled for using multiple imputation methods. ${ }^{48}$

To achieve $95 \%$ power and detect a difference in HRs of 0.5 in prostate cancer mortality for a binary risk factor using an alpha of 0.05 , a sample of at least 8762 men with prostate cancer would be required, assuming a $1 \%$ annual mortality rate over a median 10-year follow-up.

The primary outcome measure will be prostate cancer mortality. Participants will be presumed to be alive at the end of the follow-up period if they have not been reported as deceased according to the ONS mortality 
Table 1 Prognostic factors to be assessed

\begin{tabular}{|c|c|c|}
\hline Category & Prognostic factor(s) & Definition/unit \\
\hline \multirow[t]{2}{*}{ Basic demographics } & Age and date of birth & Years \\
\hline & Post code & GP practice address \\
\hline \multirow[t]{3}{*}{ Physiological } & Height & Centimetres (cm) \\
\hline & Weight & Kilograms (kg) \\
\hline & Waist:hip ratio & \\
\hline Genetic & Family history of prostate cancer & $\begin{array}{l}\text { Recorded diagnosis in first-degree } \\
\text { or second-degree relative }\end{array}$ \\
\hline \multirow[t]{6}{*}{ Biochemical } & $\begin{array}{l}\text { Triglycerides, HDL cholesterol, LDL cholesterol and VLDL } \\
\text { cholesterol }\end{array}$ & $\mathrm{mmol} / \mathrm{L}$ \\
\hline & Ferritin & $\mu g / L$ \\
\hline & Haemoglobin & $g / L$ \\
\hline & Albumin & $g / L$ \\
\hline & Serum glucose and plasma glucose & $\mathrm{mmol} / \mathrm{L}$ \\
\hline & Lead & $\mu g / L$ \\
\hline \multirow[t]{2}{*}{ Lifestyle } & Smoking history & $\begin{array}{l}\text { Smoking tobacco prior to or at index } \\
\text { date }\end{array}$ \\
\hline & Relationship status & $\begin{array}{l}\text { Patient identifies as being in a } \\
\text { relationship }\end{array}$ \\
\hline
\end{tabular}

COPD, chronic obstructive pulmonary disease; CRP, C reactive protein; GP, General Practitioners; HbA1c, haemoglobin A1c; HDL, highdensity lipoprotein; LDL, low-density lipoprotein; ONS, Office for National Statistics; PSA, Prostate Specific Antigen; VLDL, very-low-density lipoprotein.

data. Secondary outcome measures of prostate cancer progression will include all-cause mortality, change from localised to metastatic disease, and commencing antiandrogen therapy or chemotherapy. We will use whether the treatment recorded in the registry is stated to be localised (ie, one tumour treated) or systemic (ie, more than one tumour treated) to help distinguish between early and advanced disease.

Descriptive statistics will be used to summarise the basic demographic details of the men. The prevalence of the preselected putative prognostic factors will be calculated and presented. Cox proportional hazards regression will be used to estimate the crude and mutually adjusted HRs (with $95 \%$ CI) for prostate cancer and all-cause mortality according to the prognostic factors. Related prognostic factors, such as smoking and chronic obstructive pulmonary disease, will also be grouped to account for potential intermediate variables. This analysis will be repeated with stratification by stage at diagnosis. In order to allow for flexibility in the shape of the cumulative hazard function, we will use flexible parametric survival models ${ }^{49}$ for prognostic modelling. These models incorporate cubic spline terms in the log cumulative hazard function and are based on weibull, loglogistic or lognormal distributions of survival time. We will check for non-linearities in the effects of continuous predictors using fractional polynomials ${ }^{50}$ and also test for time-varying effects of prognostic factors. We will determine mortality risk in groups defined by important prognostic factors. To asses competing risks, we will use cause-specific survival analysis to estimate at 1, 2, 5 and 10 years post prostate cancer diagnosis the contribution of 
prostate cancer mortality to overall mortality in those who have died by prognostic factor combinations. ${ }^{51}$

\section{ETHICS AND DISSEMINATION}

This study protocol has approval from the Independent Scientific Advisory Committee for the UK Medicines and Healthcare products Regulatory Agency Database Research (protocol 17_041).

The findings of this study will be submitted as a manuscript to peer-reviewed journal to aid dissemination to clinicians and other researchers in the field. It will also be presented and discussed at local CPRD working groups to inform other researchers' methods using the CPRD database. Subsequent studies of the prediction tool, based on this piece of research, will involve clinicians at every stage to ensure the final tool is acceptable for use in clinical practice.

\section{CONCLUSIONS}

This study will lay the foundation for the development of a clinically useful risk prediction tool. Clinicians will be able to use the tool, inputting routine primary care data, to improve shared decision-making about an individual's prognosis and, if validated and shown effective in trials, inform their practice when deciding with patients whether to undergo radical surgery or radiotherapy or be followed up conservatively using active monitoring.

Patients will also benefit from this work in other ways. They will be able to receive more information from GPs and NHS specialists about the risk of progression of their prostate cancer, and they will be able to decide within a shared decision-making framework with their doctors about the potential benefits and harms of undergoing radical treatment or active monitoring.

Contributors SWDM: performed the literature review that informed this protocol; drafted the introduction, methods and dissemination sections. RMM and MTM: drafted the statistical analysis outline. All authors: reviewed the draft manuscript, commented and amended it, and approved the final submission.

Funding This project is in part funded by the NIHR CLAHRC West. SWDM is the recipient of an NIHR academic clinical fellowship. RMM is supported by a Cancer Research UK Programme Grant, the Integrative Cancer Epidemiology Programme (C18281/A19169).

Disclaimer The views expressed are those of the authors and not necessarily those of the NHS, the NIHR or the UK Department of Health.

Competing interests RMM has received grants from Cancer Research UK in the previous 3 years.

Patient consent Not required.

Provenance and peer review Not commissioned; externally peer reviewed.

Open Access This is an Open Access article distributed in accordance with the terms of the Creative Commons Attribution (CC BY 4.0) license, which permits others to distribute, remix, adapt and build upon this work, for commercial use, provided the original work is properly cited. See: http://creativecommons.org/ licenses/by/4.0/

(C) Article author(s) (or their employer(s) unless otherwise stated in the text of the article) 2018. All rights reserved. No commercial use is permitted unless otherwise expressly granted.
REFERENCES

1. ONS. Cancer registration statistics, England. United Kingdom: Office for National Statistics, 2014

2. ONS. Cancer survival in England: Patients diagnosed between 2010 and 2014 and followed up to 2015. United Kingdom: Office for National Statistics, 2016:1-15.

3. NICE. Suspected cancer: recognition and referral. United Kingdom: NICE, 2015:1-95.

4. Andriole GL, Crawford ED, Grubb RL, et al. Mortality results from a randomized prostate-cancer screening trial. $N$ Engl $J$ Med 2009;360:1310-9.

5. Schröder FH, Hugosson J, Roobol MJ, et al. Screening and prostate cancer mortality: results of the European Randomised Study of Screening for Prostate Cancer (ERSPC) at 13 years of follow-up. The Lancet 2014;384:2027-35.

6. Jessen K, Søndergaard J, Larsen PV, et al. Danish genera practitioners' use of prostate-specific antigen in opportunistic screening for prostate cancer: a survey comprising $174 \mathrm{GPs}$. Int $J$ Family Med 2013;2013:1-6.

7. Clements A, Watson E, Rai T, et al. The PSA testing dilemma: GPs' reports of consultations with asymptomatic men: a qualitative study. BMC Fam Pract 2007;8:35.

8. Livingston P, Cohen P, Frydenberg M, et al. Knowledge, attitudes and experience associated with testing for prostate cancer: a comparison between male doctors and men in the community. Intern Med $J$ 2002;32:215-23.

9. Drummond FJ, Carsin AE, Sharp L, et al. Factors prompting PSAtesting of asymptomatic men in a country with no guidelines: a national survey of general practitioners. BMC Fam Pract 2009;10:3.

10. Evans R, Edwards AG, Elwyn G, et al. "It's a maybe test": men's experiences of prostate specific antigen testing in primary care. $\mathrm{Br} \mathrm{J}$ Gen Pract 2007;57:303-10.

11. Grönberg $\mathrm{H}$, Adolfsson J, Aly $\mathrm{M}$, et al. Prostate cancer screening in men aged 50-69 years (STHLM3): a prospective population-based diagnostic study. Lancet Oncol 2015;16:1667-76.

12. Hamdy FC, Donovan JL, Lane JA, et al. 10-Year Outcomes after Monitoring, Surgery, or Radiotherapy for Localized Prostate Cancer. N Engl J Med 2016;375:1415-24.

13. Donovan JL, Hamdy FC, Lane JA, et al. Patient-Reported Outcomes after Monitoring, Surgery, or Radiotherapy for Prostate Cancer. $N$ Engl J Med 2016;375:1425-37.

14. Bill-Axelson A, Holmberg L, Ruutu M, et al. Radical prostatectomy versus watchful waiting in early prostate cancer. N Engl J Med 2011;364:1708-17.

15. Resnick MJ, Koyama T, Fan KH, et al. Long-term functional outcomes after treatment for localized prostate cancer. N Engl J Med 2013;368:436-45.

16. Sanda MG, Dunn RL, Michalski J, et al. Quality of life and satisfaction with outcome among prostate-cancer survivors. N Engl J Med 2008;358:1250-61.

17. Smith DP, King MT, Egger S, et al. Quality of life three years after diagnosis of localised prostate cancer: population based cohort study. BMJ 2009;339:b4817-7.

18. Wilt TJ, Brawer MK, Jones KM, et al. Radical prostatectomy versus observation for localized prostate cancer. N Engl J Med 2012;367:203-13.

19. Eisenhauer EA, Therasse P, Bogaerts J, et al. New response evaluation criteria in solid tumours: revised RECIST guideline (version 1.1). Eur J Cancer 2009;45:228-47.

20. de Camargo Cancela M, Comber H, Sharp L. Age remains the major predictor of curative treatment non-receipt for localised prostate cancer: a population-based study. Br J Cancer 2013;109:272-9.

21. Akbari MR, Wallis CJ, Toi A, et al. The impact of a BRCA2 mutation on mortality from screen-detected prostate cancer. $\mathrm{Br} \mathrm{J}$ Cancer 2014;111:1238-40.

22. Cantarutti A, Bonn SE, Adami HO, et al. Body mass index and mortality in men with prostate cancer. Prostate 2015;75:1129-36.

23. van Roermund JG, Hinnen KA, Battermann JJ, et al. Body mass index is not a prognostic marker for prostate-specific antigen failure and survival in Dutch men treated with brachytherapy. BJU Int 2010;105:42-8.

24. Chamie K, Daskivich TJ, Kwan L, et al. Comorbidities, treatment and ensuing survival in men with prostate cancer. J Gen Intern Med 2012;27:492-9.

25. Omalu $\mathrm{BI}$, Hammers JL, Parwani AV, et al. Is there an association between coronary atherosclerosis and carcinoma of the prostate in men aged 50 years and older? An autopsy and coroner based postmortem study. Niger J Clin Pract 2013;16:45-4.

26. Lee J, Giovannucci E, Jeon JY. Diabetes and mortality in patients with prostate cancer: a meta-analysis. SpringerPlus 2016;5:1-11. 
27. Ørsted DD, Bojesen SE, Nielsen SF, et al. Association of clinical benign prostate hyperplasia with prostate cancer incidence and mortality revisited: a nationwide cohort study of 3,009,258 men. Eur Urol 2011;60:691-8.

28. Tewari R, Rajender S, Natu SM, et al. Diet, obesity, and prostate health: are we missing the link? J Androl 2012;33:763-76.

29. Vance TM, Azabdaftari G, Pop EA, et al. Intake of dietary antioxidants is inversely associated with biomarkers of oxidative stress among men with prostate cancer. Br J Nutr 2016;115:68-74.

30. Goetzl MA, Van Veldhuizen PJ, Thrasher JB. Effects of soy phytoestrogens on the prostate. Prostate Cancer Prostatic Dis 2007;10:216-23.

31. Ngo TC, Lee JJ, Brooks JD, et al. Smoking and adverse outcomes at radical prostatectomy. Urol Oncol 2013;31:749-54.

32. Rohrmann S, Genkinger JM, Burke A, et al. Smoking and risk of fatal prostate cancer in a prospective U.S. study. Urology 2007;69:721-5.

33. Huncharek M, Haddock KS, Reid R, et al. Smoking as a risk factor for prostate cancer: a meta-analysis of 24 prospective cohort studies. Am J Public Health 2010;100:693-701.

34. Chavarro JE, Stampfer MJ, Li H, et al. A prospective study of polyunsaturated fatty acid levels in blood and prostate cancer risk. Cancer Epidemiol Biomarkers Prev 2007;16:1364-70.

35. Salgado-Montilla J, Soto Salgado M, Surillo Trautmann B, et al. Association of serum lipid levels and prostate cancer severity among Hispanic Puerto Rican men. Lipids Health Dis 2015;14:1-7.

36. Olivan M, Rigau M, Colás $\mathrm{E}$, et al. Simultaneous treatment with statins and aspirin reduces the risk of prostate cancer detection and tumorigenic properties in prostate cancer cell lines. Biomed Res Int 2015;2015:1-11.

37. Chan JM, Kenfield SA, Paciorek A, et al. Postdiagnostic statin use and the risk of lethal prostate cancer in the health professionals follow-up study. Cancer Epidemiol Biomarkers Prev 2015;24:1638-40.

38. Assayag J, Pollak MN, Azoulay L. Post-diagnostic use of betablockers and the risk of death in patients with prostate cancer. Eur $J$ Cancer 2014;50:2838-45.
39. Neslund-Dudas C, Kandegedara A, Kryvenko ON, et al. Prostate tissue metal levels and prostate cancer recurrence in smokers. Biol Trace Elem Res 2014;157:107-12.

40. Schiffmann J, Beyer B, Tennstedt P, et al. Oncological outcome after radical prostatectomy: marital status does not make a difference. Int J Urol 2015;22:484-9.

41. Raval AD, Thakker D, Vyas A, et al. Impact of metformin on clinical outcomes among men with prostate cancer: a systematic review and meta-analysis. Prostate Cancer Prostatic Dis 2015;18:110-21.

42. Ahn J, Peters U, Albanes D, et al. Serum vitamin D concentration and prostate cancer risk: a nested case-control study. J Natl Cancer Inst 2008;100:796-804.

43. Bai XY, Qu X, Jiang X, et al. Association between Dietary Vitamin $C$ Intake and Risk of Prostate Cancer: A Meta-analysis Involving 103,658 Subjects. J Cancer 2015;6:913-21.

44. Shang Y, Han G, Li J, et al. Vasectomy and prostate cancer risk: a meta-analysis of cohort studies. Sci Rep 2015;5:9920-5.

45. Majeed A, Car J, Sheikh A. Accuracy and completeness of electronic patient records in primary care. Fam Pract 2008;25:213-4.

46. CPRD. Welcome to the Clinical Practice Research Datalink [Internet]. https://www.cprd.com/home/ (cited 18 Sep 2016).

47. Hippisley-Cox J, Coupland C. Development and validation of risk prediction algorithms to estimate future risk of common cancers in men and women: prospective cohort study. BMJ Open 2015;5:e007825-6.

48. Azur MJ, Stuart EA, Frangakis $\mathrm{C}$, et al. Multiple imputation by chained equations: what is it and how does it work? Int $J$ Methods Psychiatr Res 2011;20:40-9.

49. Royston P, Parmar MK. Flexible parametric proportional-hazards and proportional-odds models for censored survival data, with application to prognostic modelling and estimation of treatment effects. Stat Med 2002;21:2175-97.

50. Royston P, Ambler G, Sauerbrei W. The use of fractional polynomials to model continuous risk variables in epidemiology. Int J Epidemiol 1999;28:964-74.

51. Hinchliffe SR, Lambert PC. Flexible parametric modelling of causespecific hazards to estimate cumulative incidence functions. BMC Med Res Methodol 2013;13:1-14. 